\title{
The Utility of Routine Histopathological Examination of the Appendectomy Specimens
}

\author{
Abdulkarim Hasan ${ }^{1 *}$, Khalid Mohamed Nafie ${ }^{2}$, Osama Sharafeldin Abbadi ${ }^{3}$ and Adel Abdelwahed Ibrahim ${ }^{4}$ \\ ${ }^{1}$ Department of Pathology, Faculty of medicine, Al-Azhar University, Cairo, Egypt. \\ ${ }^{2}$ Department of Laboratory \& blood bank, Prince Mishari bin Saud Hospital, Baljurashi, Saudi Arabia \\ ${ }^{3}$ Department of Biochemistry, Faculty of medicine, Omdurman Islamic University, Sudan \\ ${ }^{4}$ Department of Surgery, Faculty of Medicine, Al-Azhar University, Cairo, Egypt
}

\begin{abstract}
Background: Appendectomy is the most commonly performed surgical procedure worldwide to manage appendicitis. The practice of sending all the resected appendices for routine histopathology examination depends on the concerned clinician. This study was performed to reveal the importance of routine histopathology examination of the appendectomy specimens with recording of the occurrence and distribution of unusual pathologic findings

Methods: This was a retrospective record based comparative study performed in a single Hospital in Saudi Arabia, where the medical files of 766 patients were recruited. The appendectomy specimens were obtained either from our hospital or referred from any other hospital for histopathology examination. All the unusual diagnoses were reviewed by the histopathology author and blindly re-evaluated by other pathology consultant. Statistic analysis was performed manually.

Result: Seven hundred cases were seen within the usual histopathology scope. Unusual histopathological findings were observed in 66 cases (8.6\%): four cases of mucinous neoplasms, 20 cases of fibrous obliteration or appendiceal neuroma, 18 cases showed parasitic infestation, and 24 cases were periappendicitis without appendicular acute inflammation.

Conclusion: Routine histopathology examination of the appendix not only confirms the clinical diagnosis of acute appendicitis, but also leads to the incidental diagnosis of much unusual pathologies which could have been missed by the surgeon, and these pathologies include parasitic infestation, peri-appendicitis, neuromas and appendiceal mucinous neoplasms.
\end{abstract}

\section{Keywords: Appendicitis, Appendectomy, Histopathology.}

\section{Introduction}

Appendicitis is the inflammation of the vermiform appendix, a small anatomical entity in the large intestine of amniotes. This inflammatory condition is usually bacterial. Increased total leukocyte count characterized by neutrophilia has been reported to be associated with the diagnosis of acute appendicitis ${ }^{1}$. Intestinal parasitic diseases and neoplasms are the most common unusual histopathological findings observed in specimens after appendectomies due to any cause ${ }^{2}$. Parasitic cause differs from country to country ${ }^{3}$.

Enterobius vermicularis (pinworms, oxyuris) is the parasitic infection that affects about 200 million people all over the world. At the end of the 19th century, it was first reported that the localization of Enterobius vermicularis on the appendicular lumen causes appendicitis ${ }^{4}$.

Schistosomiasis, also known also as Bilharziasis, is a rare cause of appendicitis that is usually seen only in endemic areas where the presence of fecal contamination and the snails in source of drinking water ${ }^{5}$. Those patients with parasitic infection should also take anthelmintic treatment, as appendectomy treats only consequences but not the cause of the disease ${ }^{4}$.

Primary appendiceal neoplasms include lymphomas, mesenchymal tumors and epithelial tumors ${ }^{6}$. Primary epithelial tumors of appendix are classified as neuroendocrine tumors, mucinous tumors, and mixed endocrine and glandular tumors ${ }^{7}$.

Treatment of the mucinous tumors is based on histology and stage. Low-grade neoplasms are treated surgically in early stage disease with resection of the primary site, or peritoneal debulking and hyperthermic intraperitoneal chemotherapy (HIPEC) in patients with advanced disease stage ${ }^{8}$.

Appendectomy is the most commonly performed surgical procedure worldwide to manage appendicitis ${ }^{4}$. The practice of sending all appendices specimens for routine histopathology examination depends on the concerned clinician and is variable ${ }^{9}$. Some authors are against this policy and suggest that appendices should be sent for examination only if there is an obvious macroscopic 
abnormality at surgery 10,11 . They justify their opinion by the rarity of aberrant findings, together with the significant costs of specimen processing. Others said that histopathology examination of the appendix is necessary to confirm the diagnosis of acute appendicitis and to reveal additional findings that might not be evident clinically and intra-operatively but may change the course of further management of the patient ${ }^{12,13}$. So this stratifies the benefit of histological examination of the appendix and is likely to help in the treatment of the underlying pathology. This study is performed to reveal the importance of routine histopathology examination of the appendectomy specimens with recording of the occurrence and distribution of unusual pathologic findings with spot lighting on the clinical benefits of detecting such lesion.

\section{Materials and Methods}

This was a retrospective record based comparative study performed in Prince Mishari bin Saud Hospital in Saudi Arabia, in patients whom were diagnosed clinically with acute appendicitis and underwent appendectomy between Jun 2018 and Jan 2020.The medical files of 766 patients were recruited in this study.

The patients' gender, age, White Blood Cells (WBCs) count, and histopathology diagnosis were recorded. The appendectomy specimens were obtained either from our hospital or referred from any other hospital for histopathology examination, in either occasion cases were reported in our histopathology department. Appendectomy specimens that showed any clinical suspicion other than acute appendicitis were excluded. All the unusual diagnoses were reviewed by the histopathology author and blindly reevaluated by other pathology consultant. The reporting and classification of the neoplastic lesions was based on the classification and staging of the modified Delphi consensus process, Peritoneal Surface Oncology Group International (PSOGI) and the AJCC $8^{\text {th }}$ edition.

The ethical approval was agreed by the local ethics committee. Statistical analysis of data was performed manually.

\section{Result}

In total, 766 appendectomy specimens were received in our histopathology department during 18 months with a clinical diagnosis written on the request form as acute appendicitis. Of these, there were 444 (58\%) males and 322 (42\%) were females with male: female ratio of 1.38: 1 . The youngest patient was 4 years old and the oldest was 59 years old with mean age 17.9. Most commonly affected age group is the age between 11 years and 20 years (47.8\%). Approximately half of the cases showed WBCs count less than 11,000/ul and half of them showed more than 11,000/ul.

Different histopathology diagnoses are presented in percents in a pie chart in (figure1).

Unusual histopathological findings were observed in 66 cases $(8.6 \%)$ as follows; 4 cases of mucinous neoplasms (all of them are Low-grade appendiceal mucinous neoplasm (LAMN), 3 out of 4 are stage Tis and 1 out of 4 is T3 as the tumor reaches the mesoappendix) (figure2), 20 cases showed fibrous obliteration or appendiceal neuroma (figure 3), 18 cases showed parasitic infestation (17 patients of Enterobius vermicularis and one patient of Bilharziasis disease showing Bilharzial ova and granulomatous reaction within the wall and the surrounding fat) (figure $4 \mathrm{a} \& b$ ) and lastly 24 cases of periappendicitis (all of them are females) without appendicular acute inflammation (fiugure5).

All the studied clinicopathologic characteristics are shown in Table (1).

Table 1: Clinicopathologic characteristics of the patients in the study.

\begin{tabular}{|l|l|}
\hline Patient characteristics & \multicolumn{1}{l|}{ Result } \\
\hline All patients & $766(100 \%)$ \\
\hline Age in years & 766 \\
\hline Overall (ranged 4-59 years old with mean age = 17.9) & $108(14.1 \%)$ \\
\hline $0-10$ & $366(47.8 \%)$ \\
\hline $11-20$ & $181(23.6 \%)$ \\
\hline $21-30$ & $78(10.2 \%)$ \\
\hline $31-40$ & $12(1.6 \%)$ \\
\hline $41-50$ & $21(2.7 \%)$ \\
\hline $50-59$ & \\
\hline Sex & $444(58 \%)$ \\
\hline Male & $322(42 \%)$ \\
\hline Female & \\
\hline WBCs count & 766 \\
\hline Overall (ranged 3.65-18.8 with mean WBCs count=10.42) & \\
\hline
\end{tabular}




\begin{tabular}{|l|l|}
\hline Patient characteristics & Result \\
\hline$<11,000 /$ ul & $375(49 \%)$ \\
\hline$>11,000 /$ ul & $391(51 \%)$ \\
\hline Usual Histopathology findings & \multicolumn{2}{|l|}{} \\
\hline Overall & $700(91.4 \%)$ \\
\hline Normal or catarrhal inflammation & $82(10.7 \%)$ \\
\hline Acute appendicitis without complications & $570(74.4 \%)$ \\
\hline Acute appendicitis with complications (gangrene, perforation, abscess formation) & $48(6.3)$ \\
\hline Unusual Histopathology findings & \\
\hline Overall & $66(8.6 \%)$ \\
\hline Periappendicitis & $24(3.13 \%)$ \\
\hline Parasitic appendicitis & $18(2.34 \%)$ \\
\hline Neuroma or Fibrous obliteration & $20(2.61 \%)$ \\
\hline Mucinous neoplasm & $4(0.52 \%)$ \\
\hline
\end{tabular}

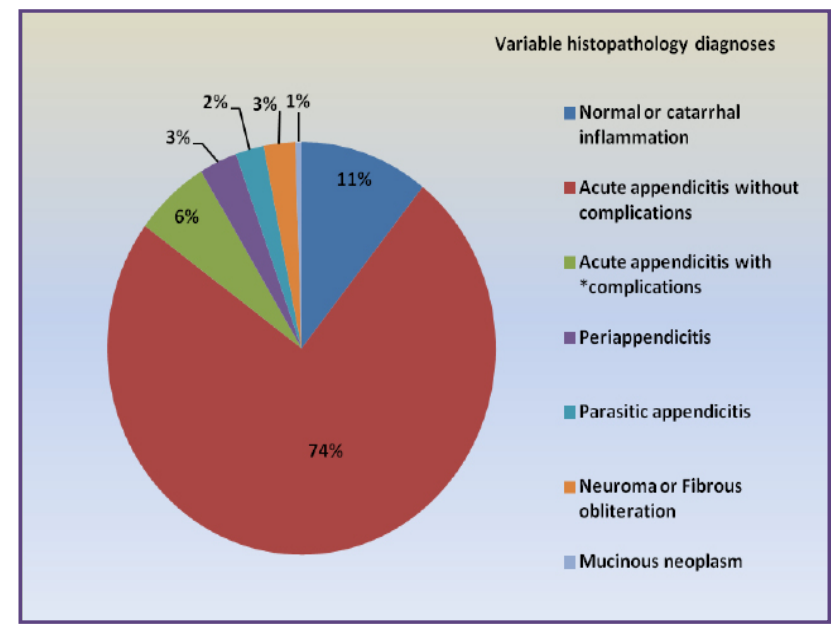

Fig. 1: A pie chart of the histopathology findings in percents. * (Complication) here is designated for gangrene, perforation, or abscess formation.

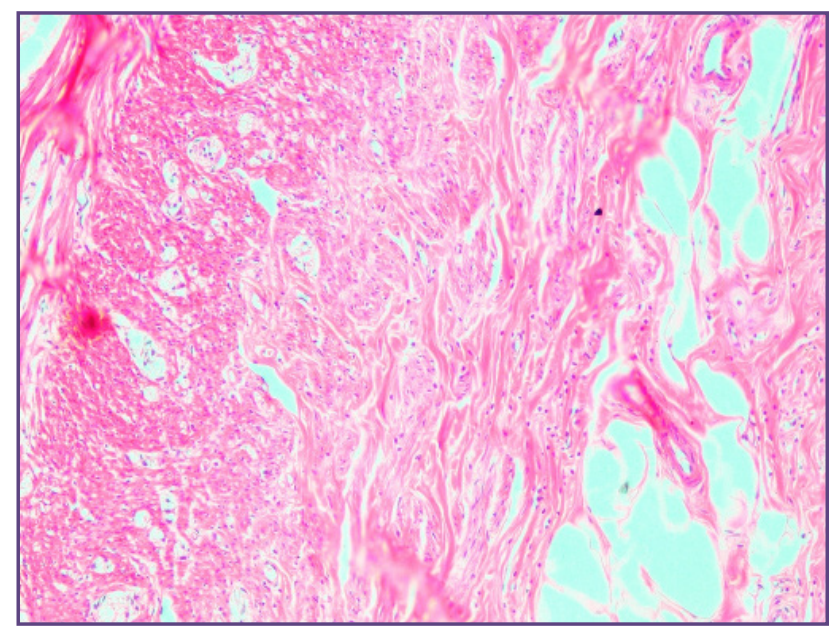

Fig. 3: Replacement of the lumen by spindle cells in fibromyxoid background with adipose tissue ( $\mathrm{H}$ and $\mathrm{E}$, $\times 10)$.

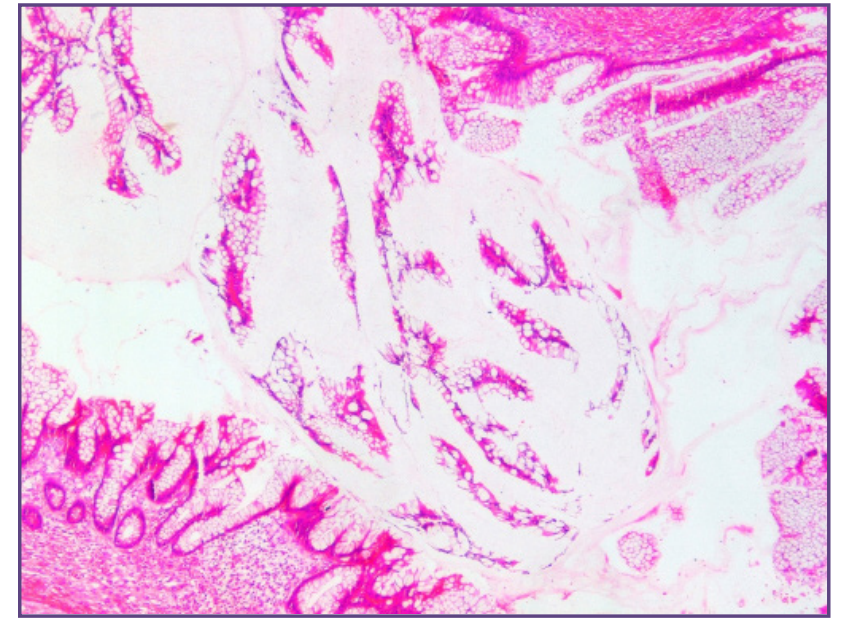

Fig. 2: Low Grade Appendiceal Mucinous Neoplasm showing mucin extravasation ( $\mathrm{H}$ and $\mathrm{E}, \times 4)$.

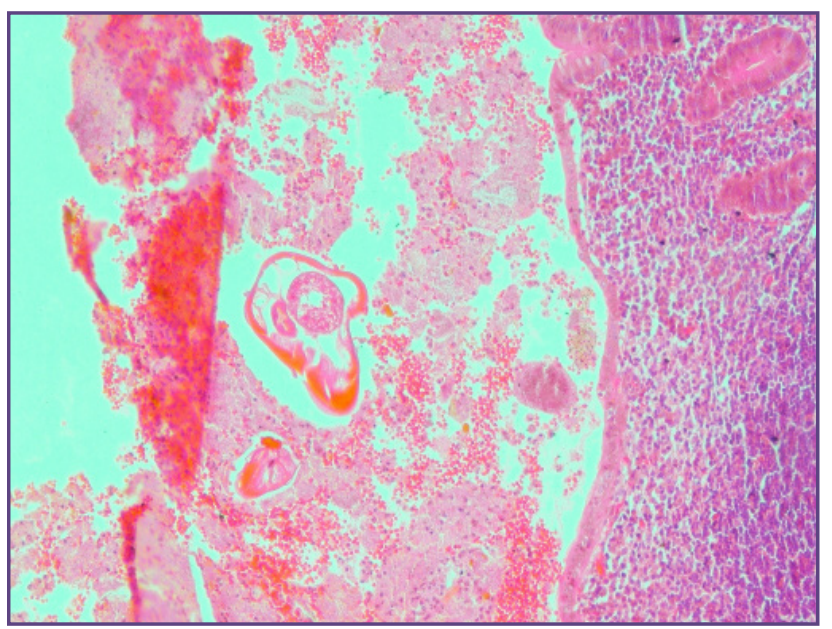

Fig. 4a: Parasitic infection; Enterobius vermicularis in the lumen of appendix ( $(\mathrm{H}$ and $\mathrm{E}, \times 10)$. 


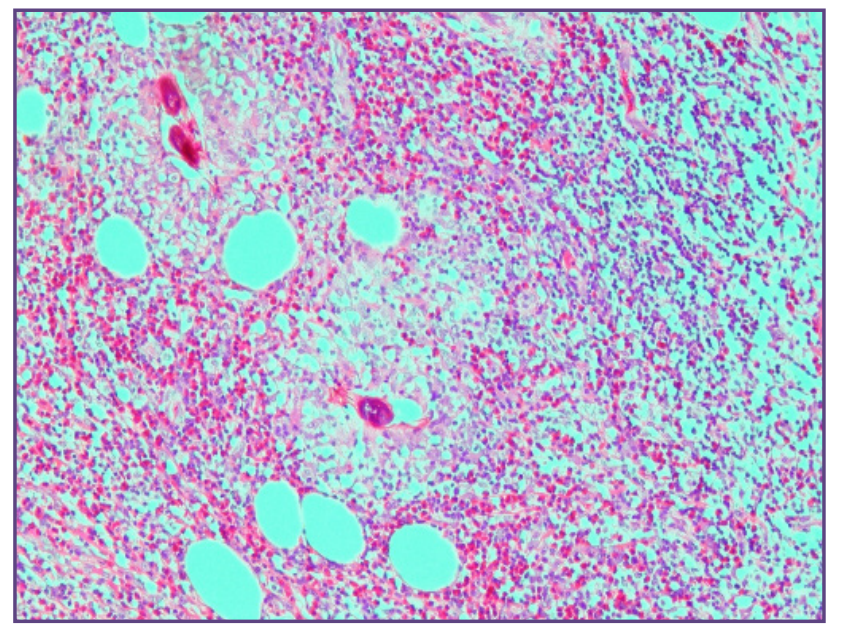

Fig. 4b: Parasitic infection; Bilharzial ova with surrounding granuloma and eosinophilic Infiltration within the appendiceal wall ( $(\mathrm{H}$ and $\mathrm{E}, \times 10)$.

\section{Discussion}

We found that the age incidence of appendectomies was higher in 10-20 years age group (47.8\%). This result is different from a study done on 325 cases in India in 2016 showed higher incidence in 20-30 years age ${ }^{10}$. A higher incidence in 20-30 years age group was also seen in a study done in Turkey by Yilmaz et al in $2013^{14}$, however another study done also in Turky in 2013 by Emre et al revealed $24.5 \%$ of all appendectomies are between 17-20 years old 15. Matariswa study (2019) showed higher incidence of appendicitis between 31 to 40 years ${ }^{16}$.

In our study, the male patients undergoing appendectomy are more than females (58\% males and 42\% females). Similar findings were shown in studies done by Dincel et al of $2018^{4}$, Rabindranath et al of (2016) ${ }^{10}$, Rehman et al of $2017^{12}$, Sharma et al of (2014) ${ }^{13}$, Yilmaz et al of (2013) ${ }^{14}$, and Chawda, Miskin, and Dombale of (2015) ${ }^{17}$. In contrast, there are two studies showed slightly female predominance, those are Shreshtha et al in $2012{ }^{18}$ and Elfaedy et al in 2019 that recorded female to male ratio of $1.1: 1^{19}$

This current study declared that $51 \%$ of all cases count were more than 11,000/ul WBCs. Rehman et al in 2017 showed $86.7 \%$ of the appendectomies are more than $11,000 / \mathrm{ul}^{12}$.

All the examined appendectomy specimens in our study were clinically diagnosed as acute appendicitis and sent to our lab for routine histopathological examination. Out of the 766 cases, 66 cases $(8.6 \%)$ showed unusual findings. This is in concordance with the results of Yilmaaz et al study in 2013 that showed 134 out of 1621 cases $(8.3 \%)$ had unusual histopathological features and slightly more than the percentages revealed by the 2013 Emre et al

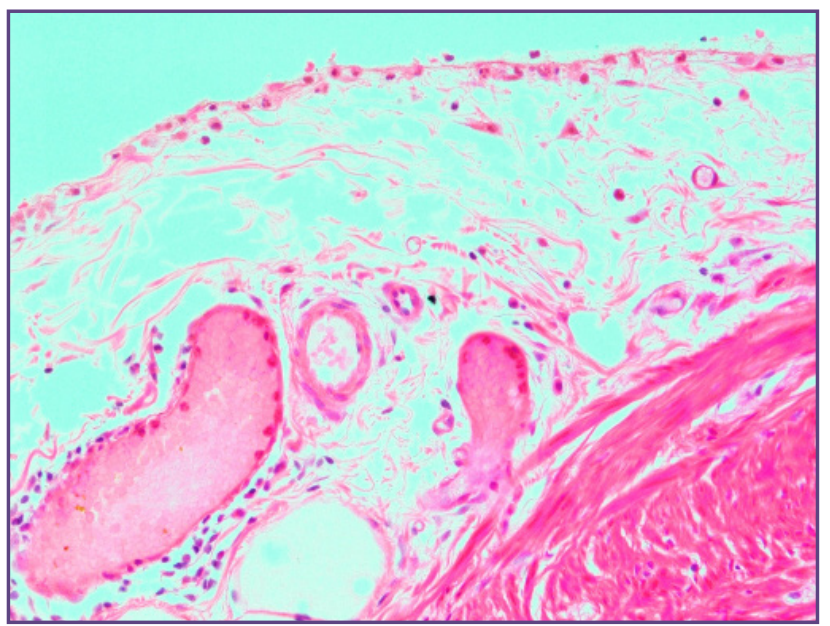

Fig. 5: Periappendicular neutrophilic infiltration ( $(\mathrm{H}$ and E, $\times 20)$.

study (7\%), and Chawda, Miskin, and Dombale study $(6.7 \%)^{17}$. Some studies showed lesser percentages of unexpected findings $(0.1-4.2 \%)$, other than normal or inflammatory appendix ${ }^{20}$. Patel and Shah in 2017 recorded $5 \%$ unusual findings ${ }^{9}$ and Dincel et al in 2018 reported 3\% unusual findings in the routinely examined appendectomy specimens ${ }^{4}$. Elfaedy et al (in 2019) ${ }^{19}$ recorded 256 out of $4012(6.2 \%)$ unusual findings but they counted the chronic appendicitis finding as an unusual, in our study we did not find any case of chronic appendicitis.

We found that 24 out of 766 cases (3.13\%) were diagnosed as periappendicitis, all of them are females, and this is to be compared with Rehman et al 2017 study that showed $56 / 316$ cases $(17.7 \%)$ were cases of periappendicitis ${ }^{12}$. Peri-appendicitis is defined as a serosal inflammation of appendix without mural or mucosal involvement ${ }^{21}$. Periappendicitis is a condition that could clinically mimics the typical presentation of appendicitis with lower right quadrant pain, fever and leukocytosis. It is primarily caused by intra-abdominal pathology; the most common etiology is acute salpingitis ${ }^{22}$. We detected two cases out of the 24 cases showing pictures of infected ovarian cysts and underwent surgical cystectomy, other two cases showed salpingitis and received medical treatment, and the rest of cases could not be reached to know the follow-up results

In our study, we found that parasitic infection represents $2.3 \%$ of all appendectomies $(2.15 \%$ are E. vermicularis and $0.15 \%$ Bilharzial infestation which highly mimics the results concluded in USA by Arca et al ${ }^{23}$ and in Iran by Ramezani and Dehghani ${ }^{24}$, who had reported E. vermicularis in $2.9 \%$ of surgically removed appendices. In Turkey, two studies recorded presence of E. vermicularis 
in $3.8 \%$ of appendectomy specimens from a Turkish population ${ }^{25,26}$. A study from Denmark reported 4\% incidence of appendiceal Entrobiasis ${ }^{27}$. A study in Cairo of Egypt done by Helmy et al ${ }^{28}$ reported that parasites were seen in $14.8 \%$ of surgically removed appendices; with approximately similar results from studies done by Jada $e t$ $a l$ in India that showed a (12\%) prevalence of parasites in appendectomy specimens ${ }^{29}$; this high incidence of parasitic infestation may be attributed to the high prevalence of these parasites in the community. Lower incidence was recorded in Nepal, where E. vermicularis was identified in only $1.62 \%$ of surgically removed appendices of Nepalese patients ${ }^{30}$.

Fibrous obliteration also known as appendiceal neuroma or neurogenous hyperplasia whereas neurogenic proliferation is identified in most of the specimens ${ }^{17}$. Therefore, various definitions including neurogenic appendicopathy and appendiceal neuroma have recently been used ${ }^{4} .20$ out of 766 cases $(2.61 \%)$ in this study were diagnosed as Neuroma or Fibrous obliteration, however in 2013 Emre et al reported that $\sim 30 \%$ of all resected appendix specimens showed fibrous obliteration. But in 2018 Dincel et al ${ }^{4}$ reported that $\sim 1 \%$ of the resected appendix specimens showed fibrous obliteration and neuroma. Neuroma of the appendix is a rare disease of neural origin that usually mimics the symptoms of the acute appendicitis ${ }^{31}$, patient's physical examination and laboratory findings can help in the differential diagnosis, but most of these cases only become evident when the histopathology examination is available ${ }^{32}$.

Histopathology examination is the key as most patients are usually detected as an incidental finding ${ }^{33}$. Regardless, the molecular mechanism underlying this pathologic process remains unknown. It is believed to occur secondary to hyperplasia of the neuroendocrine cells, because appendicular lumen replacement by and chronic inflammatory cells and fibrous tissue is generally accompanied by increased proliferation of neuroendocrine cells and nerve cells ${ }^{17}$. The importance of detecting appendiceal neuromas is that it can be a potential precursor to malignant neoplasms since the enteroendocrine cells within the nerve bundles may be hypertrophied and can be the origin of carcinoid. Complete excision of the appendix (appendectomy) is the treatment of choice ${ }^{33}$.

Appendiceal neoplasms are identified in 0.9 to $1.4 \%$ of appendiceal specimens, and the incidence is increasing, the neuroendocrine tumors (carcinoids) have long been professed as the most common neoplastic lesion of the appendix; recent data, however, has suggested a shift in epidemiology ${ }^{34}$.
In 2016 Carr et al announced that a new classification of appendiceal mucinous neoplasia is developed, and it is agreed that "mucinous adenocarcinoma" term should be reserved only for lesions with infiltrative invasion. The term "low-grade appendiceal mucinous neoplasm" is supported (It is agreed that "cystadenoma" term should no longer be recommended). The term "high-grade appendiceal mucinous neoplasm" is proposed for lesions with highgrade cytological atypia but without infiltrative invasion ${ }^{35}$.

Although a dilated, mucus-filled appendix is often called mucocele, this term is ambiguous and better to describe the imaging appearance rather than the pathologic feature ${ }^{36}$.

In our study we considered the cases with "mucocele" in clinical data as a non-routine pathology examination so we excluded those cases from our study. All the studied four cases came with clinical suspicion of acute appendicitis, two male patients and two females one of them was a pregnant woman, all of them are between 30-39 years old. After histological examination; all of them revealed features of acute inflammation along with Low-grade appendiceal mucinous neoplasm (LAMN).

As our four cases showed histological features of acute inflammation, the clinical symptoms of all those neoplastic cases were right lower abdominal pain, tenderness and vomiting and three of them showed high WBCs count (>11,000/ul). In 2018, Gündoğar et al studied 19 cases of appendiceal mucinous neoplasms revealing that the most common clinical symptoms were right lower abdominal pain $(27 \%)$, abdominal mass (16\%), weight loss (10\%), and bowel habits change in $(5 \%)$, and $(61 \%)$ of their cases presented with lower abdominal pain, whereas the rest were detected incidentally. The mean age of these 19 cases was $60 \pm 15(30-84)$ years ${ }^{37}$.

A major limitation of this study is the unavailability of patient's follow-up information especially patients with unexpected findings. Unusual findings were discovered in more than $8 \%$ of the appendectomy specimens at Histopathology examination which were not suspected in clinical assessment before surgery, intra-operatively or even on macroscopic examination but had an impact on the patient outcome. This study supports the strategy of sending all appendectomy specimens for routine histopathological examination and meticulous microscopic examination of all of those.

\section{Conclusion}

This was a retrospective record based comparative study performed in prince Mishari bin Saud Hospital in Saud Arabia, in patients whom were diagnosed clinically with acute appendicitis and underwent appendectomy between 
Jun 2018 and Jan 2020. The study concluded that routine histopathology examination of the appendix not only confirms the clinical diagnosis of acute appendicitis, but also leads to the incidental diagnosis of much unusual pathologies which could have been missed by the surgeon, and these pathologies include parasitic infestation, periappendicitis, neuromas and low grade appendiceal mucinous neoplasms. The diagnosis of the above mentioned pathologies is imperative for further patient management.

\section{Funding}

None.

\section{Competing Interests}

Authors have no competing interests to declare.

\section{Reference}

1. $\mathrm{Ng} \mathrm{KC}$, Lai SW. Clinical analysis of the related factors in acute appendicitis. Yale J Biol Med. 2002; 75(1):41-45.

2. Kozar RA, Roslyn JJ. The appendix. In: Schwartz SI. Schwartz's Principles of surgery. 7th ed. New York, NY: McGraw-Hill; 1999.

3. Al-Omran M, Mamdani MM, Mcleod RS. Epidemiologic features of acute appendicitis in Ontario, Canada. Can J Surg. 2003; 46:263-268.

4. Dincel O, Göksu M, Türk BA, Pehlivanoğlu B, İșler S. Incidental Findings in Routine Histopathological Examination of Appendectomy Specimens; Retrospective Analysis of 1970 Patients. Indian J Surg. 2018; 80(1):48-53. doi:10.1007/s12262-016-1557-z

5. Steinmann P, Keiser J, Boss R, Tanner M, Utzinger J. Schistosomiasis and water resources development: systemic review, meta-analysis and estimates of people at risk. Lancet Infect Dis. 2006; 6(7):411-425.

6. Tirumani SH, Fraser-Hill M, Auer R, Shabana W, Walsh C, Lee F, Ryan JG. Mucinous neoplasms of the appendix: a current comprehensive clinicopathologic and imaging review. Cancer Imaging. 2013; 13:14-25.

7. Odze RD, Goldblum JR. Surgical Pathology of the GI Tract, Liver, Biliary Tractand Pancreas. 3rded. Philadelphia, PA: Elsevier; 2015. pp. 779-802.

8. Shaib WL, Assi R, Shamseddine A, et al. Appendiceal Mucinous Neoplasms: Diagnosis and Management [published correction appears in Oncologist. 2018 Jan;23 (1):137]. Oncologist. 2017; 22(9):1107-1116. doi:10.1634/ theoncologist.2017-0081.

9. Patel MM, Shah RJ. Impact of Histopathological Examination of Appendix in Context to Clinical Management of Patients. Annals of Pathology and Laboratory Medicine. 2017; 4(6): 699-704.

10. Rabindranath D, Khan AA, Ansari H, Senthil P. Unusual incidental findings of routine histopathological examination of appendectomy specimens- a 2-year retrospective analysis with review of the literature. Int J of Allied Med Sci and Clin Res. 2016; 4(1):90-98.

11. Matthyssens LE, Ziol M, Barrat C, Champault GG: Routine Surgical Pathology in General Surgery. Br J Surg. 2006; 93:362-368

12. Rehman S, Khan AI, Ansari HA, Alam F, Vasenwala SM, Alam K, Khan M A. Retrospective analysis of appendicectomy specimens: A tertiary care center-based study. Saudi Surg J. 2017;5:71-5

13. Sharma S, Mahajan D, Mohsin-ul-Rasool, Bashir S, Hafiz A, Wajahat M. Histopathology of Appendicectomy Specimen: A 5 Year Study. Scholars Journal of Applied Medical Sciences. 2014; 2(1B):176-180.

14. Yilmaz M, Akbulut S, Kutluturk $\mathrm{K}$, et al. Unusual histopathological findings in appendectomy specimens from patients with suspected acute appendicitis. World J Gastroenterol. 2013; 19(25):4015-4022. doi:10.3748/wjg. v19.i25.4015

15. Emre A, Akbulut S, Bozdag Z, Yilmaz M, Kanlioz M, Emre R, et al. Routine histopathologic examination of appendectomy specimens: retrospective analysis of 1255 patients. Int Surg. 2013; 98: 354-62.

16. Matariswa S. Need of Histopathological Evaluation in Routine Appendectomy Specimens. IJPRP. 2019; 8 (6): 733738.

17. Chawda HK, Miskin AT, Dombale VD. Spectrum of histopathological lesion in surgically removed appendix. Journal of Drug Discovery and Therapeutics. 2015; 3:53-56.

18. Shreshtha R, Ranabhat SR, Tiwari M. Histopathological analysis of appendectomy specimens. J Pathol Nepal. 2012; 2: $215-9$

19. Elfaedy O, Benkhadoura M, Elshaikhy A, Elgazwi K. Impact of routine histopathological examination of appendectomy specimens on patient management: a study of 4012 appendectomy specimens. Turk J Surg. 2019; 35 (3): 196-201.

20. Gupta K, Solanki A,Vasishta RK. Appendiceal neuroma: report of an elusive neuroma. Trop Gastroenterol. 2020; $32: 332-3$.

21. Mukherjee A, Schlenker E, LaMasters T, Johnson M, Brunz J, Thomas E. Periappendicitis: Is It a Clinical Entity?. Am Surg. 2002; 68 (10), 913-6

22. Odze RD, Goldblum JR. Odze and Goldblum Surgical Pathology of the GI Tract, Liver, Biliary Tract and Pancreas, 3rd ed. Philadelphia, PA: Saunders (ELSEVIER imprint); 2014.

23. Arca MJ, Gates RL, Groner JI, Hammond S, Caniano DA. Clinical manifestations of appendiceal pinworms in children: an institutional experience and a review of the literature. Pediatr Surg Int. 2004; 20:372- 375.

24. Ramezani MA, Dehghani MR. Relationship between Enterobius vermicularis and the incidence of acute appendicitis. Southeast Asian J Trop Med Public Health. $2007 ; 38: 20-23$ 
25. Bilgin N. Appendicitis. In: Sayek İ, editor. Basic Surgery Handbook. 3rd ed. Ankara: Güneş Kitabevi; 2004. Pp.11911197. [Original language: Turkish.]

26. Engin O, Calik S,Calik B, Yildirim M, Coskun G. Parasitic appendicitis from past to present in Turkey. Iranian J Parasitol. 2010; 5:57-63.

27. Wiebe BM. Appendicitis and Enterobius vermicularis. Scand J Gastroenterol. 1991; 26:336-338.

28. Helmy A H, Abou Shousha T, Magdi M, Sabri T. Appendicitis:Appendectomy and the value of endemic parasitic infestation. Egyp J Surg. 2000; 19:87-91.

29. Jada S K, Jayakumar K, Sahu P S, Vinorth R. Faecolith examination for spectrum of parasitic association in appendicitis. J Clin Diagn Res. 2014; 8:16-18.

30. Sah SP, Bhadani PP. Enterobius vermicularis causing symptoms of appendicitis in Nepal. Trop Doct. 2006; 36:160-162.

31. Molina GA, Torres MA, Montenegro MS, Sánchez GD, Arcia AC, Enríquez JJ, Ayala AV, Orejuela ME. Neuroma of the appendix, a rare cause of appendicitis and an important reason for close follow-up. Journal of Surgical Case Reports. 2020; 3: 1-3
32. Jagtap S, Boral S, Jagtap S, Ajagekar P. Appendiceal neuroma: an uncommon entity. Surgery. 2019; 6:2631.

33. Stanley MW, Cherwitz D, Hagen K, Snover DC. Neuromas of the appendix: a light-microscopic, immunohistochemical and electron-microscopic study of 20 cases. Am J Surg Pathol. 1986; 10:801-815.

34. Hatch QM, Gilbert EW. Appendiceal Neoplasms. Clinics in Colon and Rectal Surgery. 2018; 31(5): 278-287.

35. Carr NJ, Cecil TD, Mohamed F, et al. A Consensus for Classification and Pathologic Reporting of Pseudomyxoma Peritonei and Associated Appendiceal Neoplasia: The Results of the Peritoneal Surface Oncology Group International (PSOGI) Modified Delphi Process. Am J Surg Pathol. 2016; 40(1):14-26. doi:10.1097/PAS.0000000000000535

36. Kehagias I, Zygomalas A, Markopoulos G, et al. Diagnosis and treatment of mucinous appendiceal neoplasm presented as acute appendicitis. Case Rep Oncol Med. 2016; 2016:2161952.

37. Gündoğar Ö, Kımıloğlu E, Komut N, et al. Evaluation of appendiceal mucinous neoplasms with a new classification system and literature review. Turk J Gastroenterol. 2018; 29(5):533-542. doi:10.5152/tjg.2018.17605.

*Corresponding author:

Dr. Abdulkarim Hasan, MD (Pathology), Department of Pathology, Faculty of medicine, Al-Azhar University, 11884 Cairo, Egypt.

Phone: +91966552422996

Email: doctorabdulkarim7@gmail.com

Date of Submission : 02/04/2020

Financial or other Competing Interests: None. 